\title{
Durable clinical benefit to trastuzumab and chemotherapy in a patient with metastatic colon adenocarcinoma harboring ERBB2 amplification
}

\author{
Umut Disel ${ }^{1}$, Alexis Germain ${ }^{2}$, Bahar Yilmazel ${ }^{2}$, Huseyin Abali ${ }^{3}$, Filiz Aka Bolat ${ }^{3}$, Roman \\ Yelensky ${ }^{2}$, Julia A. Elvin², Doron Lipson ${ }^{2}$, Juliann Chmielecki ${ }^{2}$, Kai Wang ${ }^{2}$, Philip J. \\ Stephens $^{2}$, Jeffrey S. Ross ${ }^{2,4}$, Vincent A. Miller ${ }^{2}$, Siraj M. Ali ${ }^{2}$, Thomas J. George Jr. ${ }^{5}$ \\ ${ }^{1}$ Adana Numune Education and Research Hospital, Department of Medical Oncology, Adana/Turkey \\ ${ }^{2}$ Foundation Medicine, Inc. Cambridge, MA, USA \\ ${ }^{3}$ Baskent University School of Medicine Adana, Turkey \\ ${ }^{4}$ Albany Medical Center, Albany, NY \\ ${ }^{5}$ University of Florida College of Medicine Gainesville, FL, USA
}

Correspondence to: Umut Disel, email: doctorhope@gmail.com

Keywords: ERBB2, HER2, trastuzumab, oxaliplatin, colorectal adenocarcinoma

Received: June 15, $2015 \quad$ Accepted: June 30, $2015 \quad$ Published: July 1, 2015

This is an open-access article distributed under the terms of the Creative Commons Attribution License, which permits unrestricted use, distribution, and reproduction in any medium, provided the original author and source are credited.

\section{ABSTRACT}

Somatic ERBB2 amplification or activating mutations occur in approximately 2-5\% of metastatic colorectal adenocarcinomas and are presumed to be oncogenic drivers, but limited evidence exists to suggest these lesions are sensitive to targeted monotherapy in patients. Here we present the case of a patient with advanced CRC with pulmonary metastases, who had progressed on both standard of care cytotoxic chemotherapy and anti-EGFR targeted therapy. Comprehensive genomic profiling (FoundationOne ${ }^{\circledR}$ ) identified amplification of ERBB2 and a $T P_{53}$ mutation in the metastatic lesion. Treatment with trastuzumab with a chemotherapy backbone elicited stable disease/minor response in the patient over a one year course of therapy, reducing tumor burden and significantly improving quality of life. This report demonstrates the application of personalized targeted therapy guided by comprehensive genomic profiling in metastatic colorectal adenocarcinoma.

\section{CASE PRESENTATION}

A 39-year old female was diagnosed with a sporadic rectal adenocarcinoma which was excised via low anterior resection (LAR), and staged through pathologic examination of the tumor as pT3N0 without infiltration of adjacent lymph nodes or distant metastasis [1]. The patient received adjuvant chemoradiotherapy followed by 6 months of infusional 5-fluorouracil (5-FU) and leucovorin chemotherapy. Approximately one year after initial diagnosis, two lesions were discovered in the lungs consistent with metastatic disease. Pulmonary metastasectomy was completed followed by a 6 month course of FOLFIRI (5-FU, leucovorin and irinotecan) and bevacizumab. One year later, identification of additional lesions in the lungs prompted a second metastasectomy followed by 6 cycles of capecitabine. Her disease continued to progress with multiple metastases to the lungs, abdomen, and pelvis. To obviate symptoms arising from a large pelvic tumor, abdomino-perineal resection was performed and subsequent molecular testing confirmed the tumor as KRAS wild-type. Anti-EGFR combination treatment with FOLFIRI and cetuximab was initiated and continued for 9 months until termination due to disease progression. Patient then received XELOX (capecitabine and oxaliplatin) chemotherapy for 7 months again until disease progression occurred. Her ECOG performance status declined to 3 due largely to cancer-related symptoms including limited mobility, dyspnea with cough, and abdominal pain. Comprehensive genomic profiling was obtained to identify rational approaches to targeted therapy in a young patient who had fully exploited guideline driven therapies. 


\section{RESULTS}

Comprehensive genomic profiling identified amplification of ERBB2 (quantitatively estimated as 21 copies) in the metastatic tumor, as well as a loss of function point mutation within $T P_{53}$. Combination therapy of trastuzumab with a backbone of capecitabine and oxaliplatin was started. The patient's performance status began to improve within two months of initiating treatment, with an ECOG score of 2, improving over the course of the next five months to zero, reflecting increased mobility and near complete resolution of symptoms. Of note, oxaliplatin was eliminated after 6 months of treatment. Imaging with PET/CT scans at 3 and 5 months post anti-ERBB2 treatment showed reduction of tumor burden with SUV in the lesion at the apex of the right lung falling from 5.1 to 4.0 and reduction of tumor volume by close to $20 \%$ (Figure 1). For the residual pelvic disease, SUV reduced from 12.2 to 9.1 with an estimated $33 \%$ reduction in metastatic disease in that area. The tumor markers CEA and CA 19-9 both showed marked declines over the first 7 months of treatment, each reduced to a third of their initial values. Treatment with trastuzumab continued for 12 months in total, after which time the patient's symptoms returned with biomarkers and radiology confirming progressive disease.

\section{METHODS}

Metastatic tumor samples from a previous pulmonary metastasectomy were submitted for histopathology, as well as comprehensive genomic profiling using the FoundationOne ${ }^{\circledR}$ assay (Foundation Medicine Inc., Cambridge, MA). Hybridization capture of 3,734 exons from 236 cancer-related

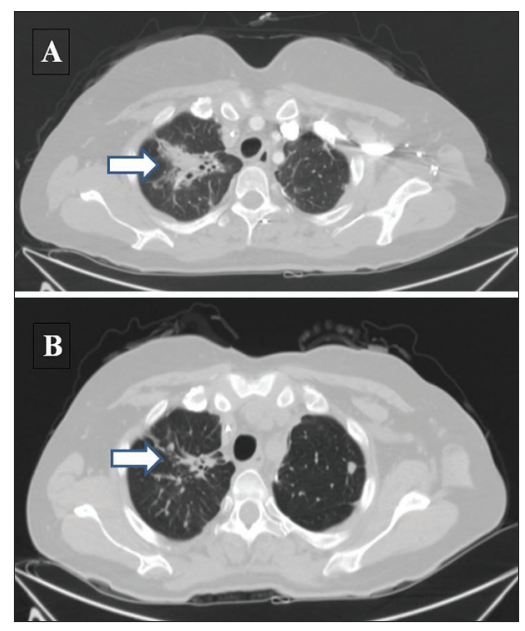

Figure 1: Representative CT scan images of upper lung metastasis at baseline (A) and after 3 months of trastuzumab and chemotherapy (B). Arrow indicates significantly regressed tumor burden. (Note: respiratory variability accounts for slight anatomic differences in pre/post imaging). genes and 47 introns of 19 genes commonly rearranged in cancer was applied to $\geq 50 \mathrm{ng}$ of DNA extracted from formalin fixed paraffin embedded tumor and sequenced to a median coverage depth of $>500 x[2]$.

\section{DISCUSSION}

Colorectal cancer affects a substantial patient population, with an estimated 132,700 incident cases in the US in 2015. CRC causes 49,700 deaths annually, making this disease the second most common cause of cancer death in men and women combined [3]. Prognosis is tightly linked to the stage at diagnosis, with patients presenting with metastatic disease surviving a median of 2 years with palliative systemic therapy [4]. Recent advances in surgical resection and combination therapy have significantly improved the 5 year survival for a subset of patients with oligometastatic disease [5]. However, for most patients with metastatic disease, medical management is oriented towards minimizing disease and treatment-associated complications in the context of disease palliation [6].

$E R B B 2$ encodes the receptor tyrosine kinase HER2, a member of the epidermal growth factor receptor family that has been linked to normal cell proliferation and tissue growth. ERBB2 amplification (2.5-3\%) or mutation ( $2 \%)$ is present in a small subset of CRC patients [7,8]. In CRC, overexpression of membranous HER2 corresponds to the observed frequency of associated gene alterations [9]. Little is known about the prognostic value of HER2 overexpression in CRC, although a recent meta-analysis found no significant effect on survival [10]. ERBB2 amplification and HER2 overexpression have best been characterized in carcinomas of the breast where it classifies $15-20 \%$ of cases as a distinct subset with poor prognosis and increased probability of recurrence [11]. Trastuzumab, an anti-HER2 monoclonal antibody that targets the extra-cellular domain, is FDAapproved for use in gastric and breast cancers overexpressing HER 2 and has shown efficacy in combination with cytotoxic chemotherapy [12,13]. Studies of ERBB2 amplification in gastric and breast cancer patients have shown a correlation between copy number alteration and sensitivity to treatment with trastuzumab, with higher copy number alteration leading to more complete responses and improved overall survival $[14,15]$.

The efficacy of trastuzumab in breast and gastric cancers suggests by analogy that patients with ERBB2 amplified CRC may also benefit from targeted anti-HER2 therapies such as trastuzumab, as in the case reported here. At least two case studies have demonstrated consistent responses of $E R B B 2$ amplified left sided colorectal carcinoma and rectal carcinoma to trastuzumab monotherapy $[16,17]$. The relatively high copy number observed in this case of rectal carcinoma may predict response to anti-HER2 targeted therapy, akin to the same phenomenon in ERBB2 amplified breast cancer, but quantitative estimates of copy number were not available for the previous cases in the literature. 
In a recent report by the HERACLES study investigators, 913 patients with metastatic CRC were screened to identify 44 with HER2 overexpression [18]. Although preliminary, treatment with trastuzumab and lapatinib in this sub group of ERBB2 amplified cancer as identified by FISH or IHC was without significant toxicity and demonstrated moderate activity. Other clinical trials targeting this population with anti-HER2 targeted therapy and a chemotherapy backbone such as NCT00006015 and NCT00003995 were initiated over a decade ago and had negative results, but enrolled either an unstratified population of advanced CRC patients, or relied on solely IHC to screen in patients [19]. The early termination of these trials due to lack of sufficient accrual has led some to propose limited applicability of anti-HER2 therapy in CRC patients due to the low frequency of HER2 overexpression [20]. However, due to the high incidence of CRC, anti-HER2 targeted therapy has the potential for use in over 3,000 patients a year in the US alone. Comprehensive genomic profiling offers both quantitative estimation of $E R B B 2$ amplification as well as assessment of the remainder of the genomic profile, and when applied in the course of clinical care could provide greater insight into the efficacy of anti-HER2 targeted therapy in ERBB2 amplified CRC patients.

The prognosis is unfavorable for patients with metastatic CRC refractory to standard treatments with median survival of approximately 6-9 months $[21,22]$. In this patient, the additional activating alteration of $T P_{53}$ also identified through comprehensive genomic profiling has been associated with poor survival [23]. This patient with an ERBB2 amplified rectal carcinoma, after exhausting prior guideline driven therapies, responded for 12 months to trastuzumab with a XELOX backbone. Thus, this case illustrates a much better than expected outcome given failure of standard treatments, and in comparison to benefit from prior lines of therapy in the same patient. In contrast to the failure of previous treatment with XELOX alone, this patient's subsequent response suggests that the efficacy was driven by the addition of trastuzumab, likely linked to the relatively high $E R B B 2$ amplification in this case quantitatively estimated to be 21 copies.

Some investigators have suggested the ratio of progression free survival on a given regimen exceeding that of a precedent one as another surrogate for biologic effect in advanced cancer [24]. The current case of a patient treated with trastuzumab and chemotherapy demonstrates a clear, durable, symptomatic, and radiographic benefit and without significant toxicity after exhaustion of standard of care therapy. Although progression ultimately occurred, the patient experienced substantial improvement in cancerassociated symptoms. This approach of comprehensive genomic profiling allows identification of clinically relevant but infrequent genomic alterations that would otherwise remain unrecognized in the context of traditional molecular testing for colorectal carcinoma. Further investigation both in the setting of clinical trials and routine clinical practice is required to elaborate on these findings.

\section{CONFLICT OF INTEREST}

AG, BY, RY, JE, DL, JC, KW, PJS, JSR, VAM and SMA are employed by and have equity interest in Foundation Medicine, Inc. TG has received non-salary research support from Bristol-Myers Squibb and Bayer Oncology. UD, HA, and FAB have nothing to declare.

\section{REFERENCES}

1. Edge SB, Compton CC. The American Joint Committee on Cancer: the 7th edition of the AJCC cancer staging manual and the future of TNM. Ann Surg Oncol. 2010 Jun;17(6):1471-4.

2. Frampton GM, Fichtenholtz A, Otto GA, Wang K, Downing SR, He J, Schnall-Levin M, White J, Sanford EM, An P, Sun J, Juhn F, Brennan K, et al. Development and validation of a clinical cancer genomic profiling test based on massively parallel DNA sequencing. Nat Biotechnol. 2013 Nov;31(11):1023-31.

3. American Cancer Society. Cancer Facts \& Figures 2015. Atlanta: American Cancer Society; 2015.

4. Stathopoulos GP. Survival of untreated advanced colorectal cancer patients. Oncology Letters. 2011;2(4):731-733.

5. Kemeny NE. Treatment of metastatic colon cancer: "the times they are A-changing." J Clin Oncol. 2013 Jun 1;31(16):1913-6.

6. Kufe DW, Holland JF, Frei E, American Cancer Society, editors. Cancer medicine 6. 6th ed. Hamilton, Ont.; Lewiston, NY: BC Decker; 2003. 2 p.

7. Marx AH, Burandt EC, Choschzick M, Simon R, Yekebas E, Kaifi JT, Mirlacher M, Atanackovic D, Bokemeyer C, Fiedler W, Terracciano L, Sauter G, Izbicki JR. Heterogenous high-level HER-2 amplification in a small subset of colorectal cancers. Hum Pathol. 2010 Nov;41(11):1577-85.

8. JS Ross, K Wang, D Khaira, A Johnson, J Chmielecki, SM Ali, JA Elvin, R Yelensky, DM Lipson, VA Miller, PJ Stephens. Comprehensive Genomic Profiling of Clinically Advanced Colorectal Carcinoma Reveals Frequent Opportunities for Targeted Therapies. ASCO. 2015.

9. Blok EJ, Kuppen PJ, van Leeuwen JE, Sier CF. Cytoplasmic Overexpression of HER2: a Key Factor in Colorectal Cancer. Clin Med Insights Oncol. 2013;7:41-51.

10. Li C, Liu D, Ye L, Huang L, Jaiswal S, Li X, Wang H, Chen L. HER-2 overexpression and survival in colorectal cancer: a meta-analysis. J Zhejiang Univ Sci B. 2014 Jun;15(6):582-9.

11. Ross JS. Breast cancer biomarkers and HER2 testing after 10 years of anti-HER2 therapy. Drug News Perspect. 2009 Mar;22(2):93-106.

12. Boekhout AH, Beijnen JH, Schellens JHM. Trastuzumab. Oncologist. 2011;16(6):800-10.

13. Gemmete JJ, Mukherji SK. Trastuzumab (herceptin). AJNR Am J Neuroradiol. 2011 Sep;32(8):1373-4.

14. Gomez-Martin C, Plaza JC, Pazo-Cid R, Salud A, Pons F, Fonseca P, Leon A, Alsina M, Visa L, Rivera F, Galan MC, Del Valle E, Vilardell F, et al. Level of HER2 gene amplification predicts response and overall survival in HER2-positive advanced gastric cancer treated with trastuzumab. J Clin Oncol. 2013 Dec 10;31(35):4445-52. 
15. Fuchs E-M, Köstler WJ, Horvat R, Hudelist G, Kubista E, Attems J, Zielinski CC, Singer CF. High-level ERBB2 gene amplification is associated with a particularly short time-tometastasis, but results in a high rate of complete response once trastuzumab-based therapy is offered in the metastatic setting. Int J Cancer. 2014 Jul 1;135(1):224-31.

16. Sorscher SM. Marked response to single agent trastuzumab in a patient with metastatic HER-2 gene amplified rectal cancer. Cancer Invest. 2011 Aug;29(7):456-9.

17. Bensch F, van Rooijen JM, Schröder CP, Reyners AKL. A 21-year-old patient with a HER2-positive colorectal cancer. Gastroenterology. 2015 Jan;148(1):20-1.

18. Siena S, Sartore-Bianchi A, Lonardi S, et al. Trastuzumab and lapatinib in HER2-amplified metastatic colorectal cancer patients (mCRC): The HERACLES trial. J Clin Oncol 33, 2015 (suppl; abstr 3508). (HERACLES Trial EudraCT 2012002128-33).

19. Clark JW, Niedzwiecki D, Hollis D, Mayer R. Phase-II trial of 5-fluororuacil (5-FU), leucovorin (LV), oxaliplatin (Ox), and trastuzumab $(\mathrm{T})$ for patients with metastatic colorectal cancer (CRC) refractory to initial therapy. Onkologie 2003; 26(suppl3):13-46

20. Ramanathan RK, Hwang JJ, Zamboni WC, Sinicrope FA, Safran H, Wong MK, Earle M, Brufsky A, Evans T, Troetschel M, Walko C, Day R, Chen HX, Finkelstein S. Low overexpression of HER-2/neu in advanced colorectal cancer limits the usefulness of trastuzumab (Herceptin) and irinotecan as therapy. A phase II trial. Cancer Invest. 2004;22(6):858-65.

21. Yoshino T, Mizunuma N, Yamazaki K, Nishina T, Komatsu Y, Baba H, Tsuji A, Yamaguchi K, Muro K, Sugimoto N, Tsuji Y, Moriwaki T, Esaki T, Hamada C, Tanase T, Ohtsu A. TAS102 monotherapy for pretreated metastatic colorectal cancer: a double-blind, randomised, placebo-controlled phase 2 trial. Lancet Oncol. 2012 Oct;13(10):993-1001.

22. Lee JJ, Kim TM, Yu SJ, Kim D-W, Joh Y, Oh D-Y, Kwon JH, Kim TY, Heo DS, Bang Y-J, Kim NK. Single-agent capecitabine in patients with metastatic colorectal cancer refractory to 5-fluorouracil/leucovorin chemotherapy. Jpn J Clin Oncol. 2004 Jul;34(7):400-4.

23. Kressner U, Inganäs M, Byding S, Blikstad I, Påhlman L, Glimelius B, Lindmark G. Prognostic value of p53 genetic changes in colorectal cancer. J Clin Oncol. 1999 Feb;17(2):593-9.

24. Shi Q, de Gramont A, Grothey A, Zalcberg J, Chibaudel B, Schmoll H-J, Seymour MT, Adams R, Saltz L, Goldberg RM, Punt CJA, Douillard J-Y, Hoff PM, et al. Individual patient data analysis of progression-free survival versus overall survival as a first-line end point for metastatic colorectal cancer in modern randomized trials: findings from the analysis and research in cancers of the digestive system database. J Clin Oncol. 2015 Jan 1;33(1):22-8. 